\title{
Version '13
}

Richtlinien der Schweizerischen Akademie der Medizinischen Wissenschaften

Zusammenarbeit

Ärzteschaft - Industrie 
"A useful criterion in determining acceptable activities and relationships is: would you be willing to have these arrangements generally known?"

"Physicians and the Pharmaceutical Industry", Guidelines of the American College of Physicians, 1990 
Richtlinien der Schweizerischen Akademie der Medizinischen Wissenschaften

\section{Zusammenarbeit Ärzteschaft - Industrie}

Vom Senat genehmigt am 29. November 2012. Diese Richtlinien ersetzen die Richtlinien der SAMW zur "Zusammenarbeit Ärzteschaft - Industrie» von 2006. Die deutsche Fassung ist die Stammversion.

0. PRÄAMBEL 2

Grundsätze 3

I. KLINISCHE FORSCHUNG 4

Einleitung 4

Richtlinien 4

II. AUS-, WEITER- UND FORTBILDUNG 8

Einleitung 8

$\begin{array}{ll}\text { Richtlinien } & 9\end{array}$

$\begin{array}{lr}\text { III. EXPERTENTÄTIGKEIT } & 12\end{array}$

Einleitung 12

$\begin{array}{ll}\text { Richtlinien } & 12\end{array}$

IV. ANNAHME VON GELD- ODER NATURALLEISTUNGEN 14

Einleitung 14

$\begin{array}{ll}\text { Richtlinien } & 14\end{array}$

V. ANHANG 15

Glossar $\quad 15$

$\begin{array}{ll}\text { Relevante Bestimmungen und Behörden } & 17\end{array}$

Hinweise zur Ausarbeitung dieser Richtlinien 20 


\section{PRÄAMBEL}

Die Zusammenarbeit von Ärztinnen und Ärzten ${ }^{1}$ mit der Industrie ist seit langem etabliert. Sie liegt grundsätzlich im Interesse einer guten Gesundheitsversorgung und trägt vielfach zu einer Mehrung des Wissens bei. Diese Zusammenarbeit kann Interessenkonflikte und Abhängigkeiten mit sich bringen oder in Ausnahmefällen zu Konflikten mit dem Gesetz führen.

Interessenkonflikte können materieller, psychologischer oder sozialer Natur sein. Sie sind nicht eine Folge eines bestimmten Handelns oder Unterlassens. Es ist dabei auch nicht entscheidend, ob sich eine Person in einer bestimmten Situation beeinflusst fühlt.

Die SAMW veröffentlichte 2002 erstmals «Empfehlungen zur Zusammenarbeit Ärzteschaft - Industrie». Sie wurden 2005 teilrevidiert und in «Richtlinien» umbenannt, die ab 2006 galten. Diese Richtlinien flossen damals in die Standesordnung der FMH ein. Ausserdem setzte die SAMW damals eine Beratende Kommission für die Zusammenarbeit Ärzteschaft-Industrie ${ }^{2}$ ein. Diese begleitete seither die praktische Anwendung und Interpretation der Richtlinien. ${ }^{3}$

In der Praxis wurden weiterer Präzisierungsbedarf und Lücken festgestellt. Daher beschloss die SAMW 2012, die Richtlinien zu revidieren. Neben der Überarbeitung und Ergänzung verschiedener Abschnitte wurde insbesondere das Kapitel «Expertentätigkeit» hinzugefügt.

Die Richtlinien gelten für die Beziehungen der Ärzteschaft mit Zulieferern auf dem Gesundheitsmarkt, d.h. insbesondere mit Unternehmen der Pharma-, der Medizinprodukte- und der IT-Industrie. Sie sollen dabei zum richtigen Umgang mit Interessenkonflikten bei der Abgeltung von Leistungen von Ärzten durch finanzielle oder anderweitige Leistungen beitragen. Die Richtlinien sollen nicht verbieten, sondern durch das Empfehlen angemessener Verhaltensweisen im beruflichen Alltag zur Objektivität und Qualität der genannten Tätigkeiten, zur Transparenz, zur Vermeidung von Abhängigkeiten und zum bewussten Umgang mit Interessenkonflikten beitragen.

1 Im Interesse der leichteren Lesbarkeit des Textes wird im Folgenden durchwegs die männliche Bezeichnung von Personen verwendet. Die entsprechenden Texte betreffen immer auch die weiblichen Angehörigen der genannten Personengruppen.

2 www.samw.ch/de/Portraet/Kommissionen/Beratende-Kommission.html

3 Swissmedic als zuständige Vollzugsbehörde publizierte in der Schweizerischen Ärztezeitung einen ergänzenden Beitrag zu ihrer Auslegung des Vorteilsverbots von Art. 33 HMG (www.saez.ch/docs/saez) archiv/de/2007/2007-39/2007-39-416.PDF). 
Die SAMW ist sich bewusst, dass solche Richtlinien nie für alle Einzelfälle direkt anwendbare Lösungen bieten können. Sie sind in der Praxis von allen Beteiligten im Sinne ihres Geistes nach bestem Wissen und Gewissen anzuwenden und einzuhalten. Deshalb sind die Ärzteschaft und Industrie als Partner aufgerufen, ihre Beziehungen in diesem wohlverstandenen Sinn zu gestalten und wo nötig zu verbessern.

\section{Grundsätze}

Entscheidend ist, dass die Beteiligten bei Interessenkonflikten nach folgenden Prinzipien vorgehen:

- Trennungsprinzip: Ärztliches Handeln insbesondere gegenüber Patienten muss von versprochenen oder erhaltenen geldwerten Leistungen oder Vorteilen unabhängig sein. Die entsprechenden Vorgänge und Abläufe sind klar voneinander zu trennen.

- Transparenzprinzip: Versprochene oder erhaltene geldwerte Leistungen oder Vorteile, insbesondere solche ohne direkte Gegenleistung, müssen offengelegt werden.

- Äquivalenzprinzip: Leistung und Gegenleistung müssen in einem angemessenen Verhältnis zueinander stehen.

- Dokumentationsprinzip: Alle Leistungen müssen schriftlich vereinbart werden. Dabei wird detailliert festgelegt, welcher Art die Leistung und das Entgelt dafür sind und welche Leistungen zu welchem Zweck konkret erbracht werden. Betreffen solche Vereinbarungen Mitarbeitende von Institutionen im Gesundheitswesen, so sind sie von deren Arbeitgeber oder Vorgesetzten zu genehmigen.

- Vier-Augen-Prinzip: Wichtige Entscheidungen sollten nicht von einer einzelnen Person getroffen werden dürfen. Ziel ist es, das Risiko von Fehlern und Missbrauch zu reduzieren. Alle Verträge und finanzielle Transaktionen werden von 2 Personen jeder Institution unterzeichnet.

- Kontentrennungsprinzip: Drittmittel für Forschung und Lehre sind jeweils separat zu führen. Alle diesbezüglichen Transaktionen müssen transparent und revisionsfähig sein.

Die Offenlegung von Interessenkonflikten bei der Zusammenarbeit von Ärzten mit der Industrie ist ein notwendiger erster Schritt zum richtigen Umgang damit. Für Ärzte in Forschung, Klinik und Praxis geht es dabei nicht nur um Rechtsfragen, sondern auch um ihre Berufsethik. Indem sich die Ärzteschaft selber Leitplanken gibt, mit denen die staatlichen Vorschriften durch eigenverantwortliche Verhaltensregeln präzisiert und ergänzt werden, unterstreicht sie ihren Willen zur Unabhängigkeit und Glaubwürdigkeit ihres Berufsstandes. 


\section{Einleitung}

Die klinische Forschung bezweckt, Erkrankungen des Menschen auf wissenschaftlicher Basis zu verstehen und dieses Wissen zur Entwicklung wirksamer Erkennungs-, Präventions- und Behandlungsmethoden praxistauglich zu machen. Die klinische Forschung ist die unabdingbare Grundlage jeglichen Fortschritts in der Medizin.

Klinische Forschung ist ein komplexer, sich über mehrere Stufen und Jahre erstreckender Prozess zur Entwicklung neuer, besserer und sicherer präventiver, diagnostischer und therapeutischer Produkte und Verfahren; sie wird an Universitäten, Kliniken, Forschungsinstitutionen und in Arztpraxen durchgeführt. Die Durchführung klinischer Forschung richtet sich nach strengen wissenschaftlichen, ethischen und rechtlichen Anforderungen, vor allem zur Gewährleistung des Schutzes der Versuchspersonen (vgl. Anhang).

Die Zusammenarbeit klinischer Forscher mit der Industrie oder mit von ihr beauftragten Forschungsinstituten ist in vielen Bereichen eine wichtige Voraussetzung für innovative Forschung. Die Aussicht, mit einem Versuch oder dessen Ergebnissen finanzielle Vorteile oder Bekanntheit zu erlangen, kann Forscher jedoch dazu verleiten, bei der Planung, Durchführung oder Auswertung eines Versuches inkorrekt zu handeln. Die zur Gewährleistung der Qualität der Forschungsvorhaben und zum Schutz der darin einbezogenen Versuchspersonen geltenden Regeln ${ }^{4}$ bedürfen deshalb der Ergänzung durch Richtlinien, die zur Objektivität der Forschung, zur Vermeidung von Abhängigkeiten und zum bewussten Umgang mit Interessenkonflikten beitragen.

\section{Richtlinien}

1. Klinische Forschung orientiert sich an wissenschaftlichen und ethischen Standards. Klinische Forschung muss den jeweils aktuellen wissenschaftlichen und ethischen Anforderungen, den gesetzlichen Vorschriften und den international anerkannten Grundsätzen der «Good Clinical Practice» (GCP) bzw. «Guten Praxis der Klinischen Versuche $»^{5}$ entsprechen. Forschende verfügen von Gesetzes wegen über eine ihrer Funktion und Verantwortung im Forschungsprojekt entsprechende GCP-Ausbildung.

4 Humanforschungsgesetz (HFG), Heilmittelgesetz (HMG), Leitlinien "Good Clinical Practice» (GCP)

5 Zusätzlich muss gemäss Art. 9 Abs. 2 Bst. IVKlin die Prüferin oder der Prüfer über die erforderliche Ausbildung oder Erfahrung in der Guten Praxis der klinischen Versuche verfügen. 


\section{Institutionen, die klinische Forschung betreiben, evaluieren regelmässig deren Qualität.}

Die wissenschaftliche Qualität klinischer Versuche ist aufgrund ihrer Originalität und Methodik sowie ihrer Resultate (einschliesslich der Offenlegung negativer Ergebnisse) zu beurteilen. Zu berücksichtigen sind dabei die Qualität der Publikation und die Bedeutung der aus der Forschung resultierenden Erkenntnisse.

\section{Alle klinischen Versuche werden in einem öffentlich zugänglichen Register erfasst.}

Die Erfassung bezweckt insbesondere,

- die korrekte und vollständige Veröffentlichung der Ergebnisse zu gewährleisten,

- dass Protokolländerungen wissenschaftlich nachvollziehbar und begründet sind, und

- nachträgliche, GCP-widrige Veränderungen am Versuchsprotokoll zu erkennen.

Dem Register sollen die relevanten Kenngrössen zu einem Versuch entnommen werden können ${ }^{6}$.

\section{Der verantwortliche Forscher und seine Mitarbeiter haben kein finanzielles Interesse am Versuch oder dessen Ergebnis.}

Die an einem Versuch beteiligten Forscher legen gegenüber der Institution, an der sie tätig sind, ihre mit dieser Beteiligung verbundenen finanziellen Interessen offen. Insbesondere dürfen der für einen Versuch verantwortliche Forscher und seine Mitarbeiter nicht gleichzeitig Inhaber, Teilhaber, Verwaltungsrat oder bedeutender Aktionär eines Unternehmens sein, welches das zu prüfende Verfahren anwendet oder das zu prüfende Produkt herstellt oder vertreibt. Begründete Ausnahmen von dieser Regelung müssen von der Institution, an der die Forscher tätig sind, bewilligt werden.

\section{Die Durchführung und Finanzierung von Versuchen werden vertraglich geregelt}

Jeder Versuch, der im Auftrag eines Dritten (der damit zum Sponsor wird) durchgeführt und von diesem finanziert wird, ist in einem schriftlichen Vertrag geregelt. Der Vertrag ist durch den verantwortlichen Forscher und, wo zutreffend, durch den zuständigen Vertreter der Institution, für die der Forscher tätig ist, sowie durch den Sponsor zu unterzeichnen.

6 Die Registrierung klinischer Versuche wird ab Inkrafttreten des Humanforschungsgesetzes samt Verordnungen dazu vorgeschrieben sein 
Im Vertrag sind festzuhalten:

- der klinische Versuch, der Gegenstand des Vertrags ist;

- die gegenseitigen Pflichten und Verantwortlichkeiten;

- die Leistungen und Gegenleistungen bei der Durchführung des Versuchs;

- die Abgeltung, wobei deren Höhe der tatsächlich erbrachten Leistung angemessen sein soll;

- der uneingeschränkte Zugang des verantwortlichen Forschers zu allen für die Durchführung des Versuchs und zum Schutz der beteiligten Versuchspersonen relevanten Daten;

- der Zugang zu den statistischen Auswertungen;

- die Pflicht, die Versuchsergebnisse zu veröffentlichen oder öffentlich zugänglich zu machen;

- die Gewährleistung der Publikationsfreiheit des Forschers;

- die Voraussetzungen, unter denen der Versuch gegebenenfalls abgebrochen werden kann oder muss;

- die Sicherstellung der Haftung bei Schäden, die aus dem klinischen Versuch entstehen können;

- die Rechte an der späteren Nutzung der Daten bzw. Versuchsergebnisse.

\section{Die Abgeltung von Versuchen, die an Institutionen durchgeführt werden, geht an institutionelle Drittmittelkonten.}

Alle von Sponsoren im Zusammenhang mit klinischen Versuchen erbrachten finanziellen Leistungen werden auf dafür bestimmte Konten verbucht. Die Institution (Universität, Departement, Klinik, Stiftung u.a.), für welche der verantwortliche Forscher tätig ist, regelt den Zugriff auf diese Konten.

\section{Bei der Publikation einer wissenschaftlichen Arbeit zeichnen diejenigen Forschenden als Autoren verantwortlich, die einen wesentlichen Beitrag dazu geleistet haben.}

In der Publikation soll als Autor genannt werden, wer an der Planung, Datensammlung, Auswertung und/oder Manuskript-Erstellung massgeblich beteiligt war. Wenn Drittpersonen (sog. Medical Writers) an der Publikation mitwirken, sind sie namentlich aufzuführen und ihre allfällige Verbindung zu einem industriellen oder andern Sponsor offen zu legen. Gefälligkeitsautorschaft (sog. «guest authors») ist nicht statthaft.

Die Mitwirkung von «Ghost Writers», die in der Publikation nicht als mitwirkende Drittpersonen aufgeführt werden, ist nicht akzeptabel. 
8. Bei der Publikation und Präsentation von Ergebnissen eines Versuchs ist dessen finanzielle oder materielle Unterstützung offen zu legen.

In den Publikationen von Versuchsergebnissen ist in einer Anmerkung oder Fussnote für die Leserschaft deutlich erkennbar zu machen, wer den Versuch finanziert hat. Bei der Vorstellung von Versuchsergebnissen an Vorträgen, Kongressen und dergleichen ist deutlich auf diese Tatsache hinzuweisen; ebenso sind allfällige Interessebindungen der Autoren offen zu legen.

9. Die Interpretation der Ergebnisse eines Versuchs muss von den Interessen desjenigen unabhängig sein, der ihn finanziell oder materiell unterstützt.

Bei der Interpretation von Versuchsergebnissen in Publikationen und bei Präsentationen sind Interessenkonflikte zu vermeiden. Der verantwortliche Forscher muss deshalb besondere Sorgfalt darauf verwenden,

- die im Versuch festgestellten erwünschten und unerwünschten Wirkungen eines Produktes oder Verfahrens tatsachengetreu zu dokumentieren und kritisch zu diskutieren;

- das Kosten-Nutzen-Verhältnis des geprüften Produktes oder Verfahrens möglichst objektiv darzustellen.

10. Forscher wirken nicht mit beim Marketing von Produkten, an deren Prüfung sie beteiligt waren.

Für einen Versuch verantwortliche oder daran beteiligte Forscher dürfen ihre Unabhängigkeit und Glaubwürdigkeit nicht in Frage stellen, indem sie sich an Marketingaktionen für das geprüfte Produkt oder Verfahren beteiligen. 


\section{Einleitung}

Der Medizin stehen immer mehr diagnostische und therapeutische Mittel zur Verfügung. Die Aus-, Weiter- und Fortbildung der Ärzte muss sich dieser Entwicklung laufend anpassen. Die Fortbildung soll den Teilnehmern objektive und ausgewogene, für die Betreuung der Patienten nützliche und notwendige Kenntnisse, Fertigkeiten und Fähigkeiten vermitteln; sie ist eine Voraussetzung für eine angemessene Ausübung der ärztlichen Tätigkeit.

Die gesetzlich vorgeschriebene Fortbildung bedeutet für die Ärzte eine erhebliche zusätzliche Leistung. In Betracht fallen der finanzielle Aufwand für die Fortbildungsveranstaltungen sowie der Arbeitszeit- und Einnahmenausfall. Die Finanzierung dieser Kosten ist weder für die Spitäler noch für die praktizierenden Ärzte sichergestellt. Neues Wissen stellt eine Bereicherung der ärztlichen Tätigkeit dar und liegt demnach im Interesse des einzelnen Arztes.

Ein bedeutender Teil der Fortbildungsveranstaltungen wird von der pharmazeutischen Industrie und der Medizinproduktebranche (in der Folge Industrie resp. Unternehmen genannt) finanziell unterstützt («gesponsert») oder auch organisiert. Dies ist für viele Ärzte und Institutionen zur Selbstverständlichkeit geworden, kann aber zu Abhängigkeiten und Interessenkonflikten führen. Deshalb sind auch für diesen Bereich Leitplanken sinnvoll.

In der medizinischen Ausbildung und in der Weiterbildung gelten in Bezug auf die Unterstützung durch die Industrie die gleichen Überlegungen wie bei der Fortbildung. 


\section{Richtlinien}

\section{Den Antrag auf Anerkennung einer Fortbildungsveranstaltung bei den zu- ständigen Organen (Fachgesellschaften, kantonale Ärztegesellschaften, SIWF) stellen die veranstaltenden Ärzte oder die ärztlichen Fachgremien.}

Es ist Aufgabe des Veranstalters, die Anerkennung der Fortbildung bei der zuständigen Fachgesellschaft zu beantragen. Eine Anerkennung wird nur für Fortbildungen gewährt, die den vorliegenden Richtlinien vollumfänglich genügen. Veranstaltungen orientieren sich an den Zielen der Fortbildungsordnung (FBO) ${ }^{7}$ des Schweizerischen Instituts für ärztliche Weiter- und Fortbildung (SIWF) ${ }^{8}$ sowie den Fortbildungsprogrammen der Fachgesellschaften.

\section{Fortbildungsveranstaltungen werden nur anerkannt, wenn Inhalt und Ablauf} durch Ärzte bzw. ärztliche Fachgremien bestimmt oder entscheidend mitbestimmt werden.

Dafür gelten namentlich folgende Bedingungen:

- Veranstalter sind im jeweiligen Fachgebiet kompetente Organisationen, Institutionen oder Personen und nicht die Industrie.

- Fortbildungsveranstaltungen sollten durch die Teilnehmerbeiträge und die veranstaltende Institution finanziert werden. Bedarf es weiterer finanzieller Unterstützung durch Sponsoren, so sind dafür mehrere, von einander unabhängige Unternehmen vorzusehen.

- Es wird in der Regel eine Teilnahmegebühr erhoben. Bei kürzeren (halbtägigen) Fortbildungsveranstaltungen kann darauf verzichtet werden.

- Die Vereinbarungen zwischen Veranstalter und Sponsoren sind schriftlich festgehalten.

- Die Veranstalter und nicht die Sponsoren bestimmen das Programm (Inhalt und Ablauf) und wählen die Referenten aus. Von Sponsoren veranstaltete SatellitenSymposien sind als solche zu bezeichnen, auf Randzeiten zu legen, und werden nicht als Fortbildung anerkannt.

- Die Teilnehmer sollen Gelegenheit haben, Fortbildungsveranstaltungen zu evaluieren.

- Ein allfälliges Rahmenprogramm ist von deutlich untergeordneter Bedeutung. Rahmenprogramm und Fachteil müssen klar getrennt sein.

- Die Zusage von Credits für eine Fortbildungsveranstaltung muss vor dem Versand der Einladung dazu geklärt sein. Einladungen zu Fortbildungsveranstaltungen mit dem Hinweisen wie «Credits beantragt» sind nicht zulässig. Die Beantwortung von Credits-Anfragen durch die zuständigen Organe sollte innerhalb von vier Wochen erfolgen.

7 www.fmh.ch/files/pdf6/fbo_d.pdf

8 www.fmh.ch/bildung-siwf.html 
Zur Vermeidung administrativer Umtriebe können die Fachgesellschaften regelmässig durchgeführte eigene Fortbildungsveranstaltungen oder solche von Spitälern oder Spitalabteilungen en bloc oder im Voraus anerkennen; Voraussetzung dafür ist die schriftliche Zusicherung der betreffenden Fachgesellschaft oder der Spitäler und Spitalabteilungen, dass diese Fortbildungsveranstaltungen den Anforderungen der vorliegenden Richtlinien entsprechen.

3. Die Möglichkeiten der Prävention, Diagnose und Therapie werden grundsätzlich nach den Kriterien der evidenz-basierten Medizin (EBM) und unter Berücksichtigung ihrer Wirtschaftlichkeit dargestellt.

Die Themen sollen objektiv nach dem aktuellen Stand der wissenschaftlichen Erkenntnis und von verschiedenen Seiten her (interdisziplinär) behandelt werden. Die Diagnose- und Therapiemöglichkeiten sollen vollständig und grundsätzlich nach den Kriterien der EBM dargestellt werden.

4. Stehen für die besprochene Prävention, Diagnose oder Therapie mehrere wirksame Arzneimittel, Medizinprodukte oder Verfahren zur Verfügung, so ist ein objektiver Vergleich anzustreben.

In den Referaten werden Arzneimittel grundsätzlich mit der international anerkannten Wirkstoffbezeichnung ${ }^{9}$ erwähnt.

5. Finanzielle Mittel aus dem Sponsoring werden auf ein dafür bestimmtes Konto des Veranstalters (Universität, Institution, Stiftung, Fachgesellschaft, regionale Ärztevereinigung usw.) verbucht und für die Organisation von Fortbildungsveranstaltungen, Honorierung der Referenten und deren Spesen verwendet.

In Spitälern stattfindende ganz- oder mehrtägige Fortbildungsveranstaltungen, die von der Industrie unterstützt werden, sind von der dafür zuständigen Stelle zu genehmigen.

Die Kontrolle der Finanzen ist Sache der Veranstalter. Den Sponsoren und den Fachgesellschaften sind Budget und Rechnung auf Anfrage vorzulegen.

6. Die an Fortbildungsveranstaltungen als Zuhörer (d.h. ohne Präsentation, Poster, Referat, Sitzungsleitung o.ä.) teilnehmenden Ärzte leisten eine angemessene Kostenbeteiligung.

Im Interesse ihrer Unabhängigkeit bezahlen die Teilnehmer einer Fortbildungsveranstaltung oder deren Arbeitgeber einen angemessenen Beitrag an die Kosten für Teilnahmegebühr, Reise und Unterkunft, d.h. in der Regel mindestens ein Drittel dieser Kosten.

9 International Nonproprietary Names for pharmaceutical substances (INN) / Dénominations communes internationales des Substances pharmaceutiques (DCI) (www.who.int/medicines/services/inn/en/) 
Die ganze oder teilweise Rückerstattung der Kostenbeteiligung und/oder eine Vergütung der indirekten Kosten eines Teilnehmers (Arbeitszeit- oder Einkommensausfall) durch einen Sponsor sind nicht zulässig.

Angestellte Ärzte, deren Teilnahme an einer Veranstaltung ein Unternehmen finanziell unterstützen will, informieren ihre vorgesetzte Stelle über den Umfang der Unterstützung und den Sponsor. Bei Ärzten in Weiterbildung ergeht die Einladung in der Regel an die Institution, und diese entscheidet über die Teilnahme.

Die Kosten für zusätzliche Hotelaufenthalte, Reisen oder andere Aktivitäten, die mit der Veranstaltung keinen inhaltlichen Zusammenhang haben, gehen vollumfänglich zulasten der Teilnehmer bzw. allfälliger Begleitpersonen.

7. Referenten und Organisatoren legen allfällige persönliche oder institutionelle kommerzielle Interessen, finanzielle Verbindungen zum Sponsor, Beratertätigkeit im Auftrag des Sponsors oder Forschungsunterstützung durch den Sponsor offen.

Referentenhonorare sollen angemessen sein.

Im Programm und in den Unterlagen einer Veranstaltung werden alle Sponsoren aufgeführt.

Referenten legen ihre Interessenbindungen dem Veranstalter, der Fachgesellschaft sowie vor Beginn ihrer Präsentation den Teilnehmern auf geeignete Weise offen.

8. Schaffen Medizinische Fakultäten bzw. deren Universitäten eine Lehr- und/ oder Forschungsstelle (Professur), die durch Unternehmen oder andere Drittmittel finanziert wird, so bestimmen sie schriftlich die Rahmenbedingungen dafür.

Dabei ist die Unabhängigkeit von Lehre und Forschung zu gewährleisten.

9. Die Medizinischen Fakultäten sorgen dafür, dass unangemessene Interaktionen zwischen Medizinstudierenden und Industrie-Unternehmen unterbleiben.

Die Fakultäten achten insbesondere darauf, dass Studierende während ihrer Ausbildung und im weiteren Zusammenhang damit nicht von Industrieunternehmen mit Geschenken, anderweitigen geldwerten Vorteilen oder sonst in ungebührlicher Weise beeinflusst werden. Zudem sensibilisieren sie die Studierenden für mögliche Interessenkonflikte bei der Zusammenarbeit Ärzteschaft-Industrie.

10. Die Kaderärzte von Spitälern achten darauf, dass Kontakte von Industrievertretern mit Spitalpersonal in einem institutionellen Rahmen stattfinden.

Kontakte zwischen Industrievertretern und Spitalpersonal, insbesondere Assistenzärzten, sollen in der Regel in den Räumen des Spitals stattfinden. Die Kaderärzte achten darauf, über solche Kontakte und deren Inhalt informiert zu werden. 


\section{Einleitung}

Ärzte werden beigezogen, wenn es spezifische medizinische Fragen zu bearbeiten gibt, zu deren Beantwortung ihre Expertise unerlässlich ist. Die entsprechenden Anfragen stammen von unterschiedlichen Seiten. Beispiele dafür sind folgende: Eine staatliche Behörde will eine Empfehlung zum Gesundheitsverhalten veröffentlichen; ein Industrieunternehmen will eine Forschungsfrage bearbeiten oder ein neues Produkt lancieren; oder eine Fachgesellschaft will Guidelines ausarbeiten. Dabei können immer Interessenkonflikte entstehen.

\section{Richtlinien}

1. Im Hinblick auf die Mitarbeit in einem Advisory Board (oder einem ähnlichen Gremium, siehe Glossar) sollen Bedarf und Begründung für eine solche Beratungstätigkeit geklärt werden.

$\mathrm{Zu}$ prüfen ist namentlich:

- ob der Zweck der Beratung klar umschrieben und gerechtfertigt ist; zu vermeiden sind namentlich Advisory Boards für Marketingzwecke;

- Dauer und Begründung der Beratungstätigkeit;

- ob die eigene fachliche Kompetenz hinreicht, um sich zum Beratungsgegenstand glaubwürdig äussern zu können;

- ob Interessenkonflikte bestehen;

- aufgrund welcher Kriterien die Auswahl von Experten (inkl. Anzahl) erfolgt.

Gegebenenfalls ist auf die Teilnahme an einem Advisory Board zu verzichten.

2. Eine Beratungsleistung erfolgt grundsätzlich auf Basis eines Vertrags, der insbesondere Art, Zweck und Umfang der Beratungsleistung, das Honorar, die Unabhängigkeit des Experten sowie Transparenzbestimmungen dokumentiert.

3. Die Höhe des Honorars, die für die Tätigkeit in einem Advisory Board oder ähnlichen Gremium vereinbart wird, soll der erbrachten Leistung entsprechen.

4. Mitglieder von Gremien, die für die Ausarbeitung von Guidelines oder Leitlinien zuständig sind, legen zu Beginn und danach periodisch ihre Interessenkonflikte offen; diese Angaben werden zusammen mit den Guidelines oder Leitlinien veröffentlicht. 
5. Ein Arzt beteiligt sich an einer Beobachtungsstudie oder an einer OnlineBefragung nur, wenn dabei eine relevante wissenschaftliche Fragestellung bearbeitet wird und es sich nicht um eine Form von Marketing handelt.

6. Mitglieder von institutionsinternen Gremien, die für den Einkauf von Heilmitteln zuständig sind, müssen ihre Interessenbindungen offenlegen.

Bei absehbaren Interessenkonflikten soll das betreffende Mitglied am Entscheid nicht mitwirken.

7. Experten und "Opinion Leaders» lassen sich nicht als Autoren auf Publikationen setzen, an denen sie nicht massgeblich beteiligt waren und für deren Inhalt sie nicht vollumfänglich bürgen können (keine sog. "guest authors»). 


\section{Einleitung}

Artikel 38 der FMH-Standesordnung hält fest, dass «die Annahme von Geschenken [...] oder von anderen Vorteilen [...] von Dritten, die den Arzt oder die Ärztin in ihren ärztlichen Entscheidungen beeinflussen können und das übliche Mass kleiner Anerkennungen übersteigen, [...] unzulässig» ist.

Auch der Gesetzgeber hat in diesem Zusammenhang in verschiedenen Gesetzen Bestimmungen erlassen (Art.33 Heilmittelgesetz, Art. 56 Abs. 3 Krankenversicherungsgesetz, Art. 322ter ff. Strafgesetzbuch; kantonale Bestimmungen). Die folgenden Richtlinien sind als Umsetzungshilfe für die Praxis zu verstehen und zu beachten.

\section{Richtlinien}

\section{1. Ärzte in Klinik, Praxis und Forschung nehmen von der Industrie keine Geld-} oder Naturalleistungen entgegen, die das Mass finanziell unbedeutender kleiner Anerkennungen übersteigen.

An öffentlichen Spitälern ordnen interne Regeln die Entgegennahme von Geldoder Naturalleistungen. Sie bestimmen innerhalb der Institution, welche Zuwendungen von der vorgesetzten Stelle zu genehmigen sind und welche ihr nur zu melden sind (z.B. durch Bezeichnung von Obergrenzen oder durch Erstellen einer «Positivliste»).

Bei allen grösseren Einkäufen und Aufträgen braucht es eine Kollektivunterschrift (Vier-Augen-Prinzip). Die Annahme von Geld- und Naturalleistungen und das Einkaufswesen der Institution sind strikte zu trennen.

Alle Vereinbarungen über die Entgegennahme von Geld- oder Naturalleistungen oberhalb einer institutionsintern festgelegten Grenze haben schriftlich zu erfolgen. Diese Vereinbarungen enthalten auch die Zusicherung, dass keine (mündlichen oder stillschweigenden) Nebenabsprachen getroffen wurden. Zusätzlich werden auch die erlaubten Verwendungszwecke der auf dem Spendenkonto einbezahlten Gelder festgelegt. Das Verfügungsrecht über das Konto ist institutionsintern zu regeln.

\section{2. Ärzte gehen mit Gratismustern korrekt und zweckentsprechend um.}

Ärzte sollen sich bewusst sein, dass Arzneimittelmuster das Verschreibungsverhalten beeinflussen. 


\section{ANHANG}

\section{Glossar}

\section{Advisory Board}

Aus Ärzten und andern Fachleuten bestehendes Gremium, das ein Unternehmen oder eine andere Organisation im Zusammenhang mit medizinischen Fragen berät. Andere Bezeichnungen dafür sind u.a. Concept Board, Expert Panel, Executive Council oder Round Table.

\section{Arzneimittel}

Produkte chemischen oder biologischen Ursprungs, die zur medizinischen Einwirkung auf den menschlichen oder tierischen Organismus bestimmt sind oder angepriesen werden, insbesondere zur Erkennung, Verhütung oder Behandlung von Krankheiten, Verletzungen und Behinderungen; zu den Arzneimitteln gehören auch Blut und Blutprodukte (Art. 4 Abs. 1 Bst. a $\mathrm{HMG}$ ).

\section{Ausbildung}

Universitäre Grundausbildung (Studium)

\section{Drittmittel}

Finanzielle Unterstützung, die einer Person oder einer Institution von externer Stelle unter Vereinbarung einer direkten Gegenleistung (zweckgebunden, Projektfinanzierung) zur Verfügung gestellt wird und die für beide Parteien denselben Wert besitzt (im Gegensatz zu Sponsoring).

\section{Fortbildung}

Kontinuierliche Aufdatierung und Erweiterung der beruflichen Qualifikation nach abgeschlossener Weiterbildung; sie hat das Ziel, die Qualität der Berufsausübung zu sichern.

\section{Fortbildungsveranstaltung}

z.B. Kongress, Tagung, Treffen von Ärzten zum Erfahrungsaustausch («Qualitätskränzli»), Internetbasiertes Fortbildungsangebot.

\section{GCP}

Good Clinical Practice; Leitlinien zur "Guten Praxis der klinischen Versuche», mit denen einerseits der Schutz der Versuchspersonen sichergestellt und andererseits die Qualität der Ergebnisse gewährleistet werden soll.

\section{Generic Name}

International anerkannte Wirkstoffbezeichnung (International Nonproprietary Name [INN]; Dénomination commune internationale [DCI])

\section{Geschenke}

Zuwendungen ohne Leistungsvereinbarung und ohne Zweckbestimmung

\section{Heilmittel}

Oberbegriff für Arzneimittel und Medizinprodukte

\section{HFG}

Bundesgesetz über die Forschung am Menschen (Humanforschungsgesetz)

\section{Institution}

z.B. Universität, Spital, Netzwerk 


\section{Klinische Forschung}

Forschung mit Versuchspersonen oder mit Material menschlichen Ursprungs; der Forscher hat also dabei (im Gegensatz zur Grundlagenforschung) Kontakt mit Menschen. Zur klinischen Forschung zählen u.a. patienten-orientierte Forschung, epidemiologische Studien, OutcomeForschung sowie Versorgungsforschung.

\section{Klinischer Versuch}

Forschungsprojekt mit Personen, das diese prospektiv einer gesundheitsbezogenen Intervention zuordnet, um deren Wirkungen auf die Gesundheit oder auf den Aufbau und die Funktion des menschlichen Körpers zu untersuchen.

\section{Medizinprodukte}

Produkte, einschliesslich Instrumente, Apparate, In-vitro-Diagnostika, Software und andere Gegenstände oder Stoffe, die für die medizinische Verwendung bestimmt sind oder angepriesen werden und deren Hauptwirkung nicht durch ein Arzneimittel erreicht wird (Art. 4 Abs. 1 Bst. b $\mathrm{HMG}$ ).

\section{Sponsor}

Person oder Organisation, die für die Einleitung, das Management oder die Finanzierung eines klinischen Versuchs die Verantwortung übernimmt (Art. 5 Bst. b VKlin).

\section{Sponsoring}

Finanzielle Unterstützung einer Veranstaltung, eines Projektes, einer Publikation oder anderer Leistungen ohne direkte gleichwertige Gegenleistung, jedoch mit Zweckbestimmung, mit oder ohne Auflagen zur Deklaration.

\section{Versuchsperson}

Personen, die an einem klinischen Versuch teilnehmen und bei denen entweder das zu prüfende Heilmittel angewendet wird oder die einer Kontrollgruppe zugeteilt sind (Art. 5 Bst. d VKlin).

\section{Weiterbildung}

Eine bezüglich Dauer und Inhalt gegliederte, evaluierbare Tätigkeit, mit der die erworbenen Kenntnisse, Fertigkeiten und Fähigkeiten im Hinblick auf eine selbständige Berufsausübung vertieft und erweitert werden sollen und die sich an das Studium anschliesst. 


\section{Relevante Bestimmungen und Behörden}

\section{Ad I. Klinische Forschung}

\section{Nationale und internationale Regeln für die Durchführung klinischer Versuche}

Wissenschaftliche Integrität. Grundsätze und Verfahrensregeln. Akademien der Wissenschaften Schweiz, 2008.

www.akademien-schweiz.ch/index/Portrait/Kommissionen-AG/WissenschaftlicheIntegritaet.html

Deklaration des Weltärztebundes von Helsinki. «Ethische Grundsätze für die medizinische Forschung am Menschen» (revidierte Fassung Oktober 2008)

Originaltext: www.wma.net/en/30publications/10policies/b3/

deutsch: www.bundesaerztekammer.de/downloads/deklhelsinki2008.pdf

Guideline for Good Clinical Practice, International Conference on Harmonisation of Technical Requirements for Registration of Pharmaceuticals for Human Use (ICH)

Originaltext: www.ich.org/fileadmin/Public_Web_Site/ICH_Products/Guidelines/Efficacy/E6_R1/

Step4/E6_R1_Guideline.pdf

Übereinkommen zum Schutz der Menschenrechte und der Menschenwürde im Hinblick auf die Anwendung von Biologie und Medizin (Bioethik-Konvention)

Originaltext: conventions.coe.int/treaty/en/treaties/html/164.htm

deutsch: www.ruhr-uni-bochum.de/zme/Europarat.htm\#dt-0298

The CONSORT statement. Revised recommendations for improving the quality of reports of parallel-group randomised trials. The Lancet 2001; 357: 1191-1194

International Committee of Medical Journals Editors. Uniform requirements for manuscripts submitted to biomedical journals. New England Journal of Medicine 1997; 336: 309 - 315

Clinical Trial Registration. A Statement from the International Committee of Medical Journal Editors. Editorial. Annals of Internal Medicine 2004; 141: 477- 478 


\section{Arzneimittel-Zulassungsbehörden, Gesetze und weitere Vorschriften}

\section{Schweiz}

Schweizerisches Heilmittelinstitut, Swissmedic

www.swissmedic.ch

Bundesgesetz über die Arzneimittel und Medizinprodukte, Heilmittelgesetz (HMG)

www.admin.ch/ch/d/sr/c812_21.html

Verordnung vom 17. Oktober 2001 über klinische Versuche mit Heilmitteln (VKlin) www.admin.ch/ch/d/sr/c812_214_2.html

Verordnung vom 17. Oktober 2001 über die Arzneimittelwerbung (AWV) www.admin.ch/ch/d/sr/c812_212_5.html

Bundesgesetz über die Forschung am Menschen (vom Parlament verabschiedet am 30. 9. 2011; tritt voraussichtlich Anfang 2014 in Kraft)

\section{Europäische Union}

European Medical Evaluation Agency, EMA

www.ema.europa.eu/ema/index.jsp?curl=pages/home/Home_Page.jsp\&mid=

Richtlinie 2001/20/EG des Europäischen Parlaments und des Rates vom 4. Oktober 2001

zur Angleichung der Rechts- und Verwaltungsvorschriften der Mitgliedstaaten über die

Anwendung der guten klinischen Praxis bei der Durchführung von klinischen Prüfungen mit

Humanarzneimitteln

Übersicht: ec.europa.eu/health/documents/eudralex/vol-1/index_en.htm

deutsch: ec.europa.eu/health/files/eudralex/vol-1/dir_2001_83_cons2009/2001_83_

cons2009_de.pdf

\section{USA}

Federal Drug Administration, FDA

www.fda.gov/

Good Clinical Practice in FDA Regulated Clinical Trials

www.fda.gov/oc/gcp/default.htm

\section{Kodizes der Industrie}

Verhaltenskodex der pharmazeutischen Industrie in der Schweiz (Pharmakodex) vom

4. Dezember 2003 (mit nachträglichen Änderungen)

www.sgci.ch/plugin/template/sgci/*/11386

Kodex der FASMED (Medizinprodukteindustrie)

www.fasmed.ch/fileadmin/pdf/polit_dossiers/Medien/FASMED\%20CBC\%2026.05.2010.pdf

EFPIA Code of Practice on the promotion of prescription-only medicines to, and interactions with, healthcare professionals

www.efpia.eu/content/default.asp?PageID=559\&DoclD=11731

Eucomed Guidelines on Interactions with Healthcare Professionals

www.eucomed.org/key-themes/ethics 


\section{Ad II. Aus-, Weiter- und Fortbildung}

\section{Internationale Empfehlungen und Richtlinien}

World Medical Association (WMA). Statement concerning the Relationship between Physicians and Commercial Enterprises. 2009.

www.wma.net/en/30publications/10policies/r2/

Canadian Medical Association. CMA Policy. Physicians and the Pharmaceutical Industry. Update 2001.

www.cma.ca

Physician-Industry Relations

Part 1: Individual Physicians. Ann Int Med; 2002, 136: 396, Physician-Industry Relations.

Part 2: Organizational Issues. Ann Int Med 2002; 136: 403

\section{Empfehlungen der Arzneimittel-Zulassungsbehörde}

Zum Verbot des Versprechens und Annehmens geldwerter Vorteile gemäss Artikel 33 des Heilmittelgesetzes insb. in Zusammenhang mit der Unterstützung der Weiter- und Fortbildung von Medizinalpersonen durch die Pharmaindustrie. www.swissmedic.ch/marktueberwachung/00091/00241/01468/index.html?lang=de

\section{Kodizes der Industrie}

Verhaltenskodex der pharmazeutischen Industrie in der Schweiz (Pharmakodex) vom 4. Dezember 2003 (mit nachträglichen Änderungen) www.sgci.ch/plugin/template/sgci/*/11386

Kodex der FASMED (Medizinprodukteindustrie) www.fasmed.ch/fileadmin/pdf/polit_dossiers/Medien/FASMED\%20CBC\%2026.05.2010.pdf

EFPIA Code of Practice on the promotion of prescription-only medicines to, and interactions with, healthcare professionals www.efpia.eu/content/default.asp?PagelD $=559 \& D$ oc $\mid D=11731$

Eucomed Guidelines on Interactions with Healthcare Professionals www.eucomed.org/key-themes/ethics

\section{Ad III. Annahme von Geld- oder Naturalleistungen}

\section{Relevante Gesetzestexte}

Art. 33 Bundesgesetz über Arzneimittel und Medizinprodukte vom 12.12.2000 (HMG)

Art 322ter ff. Schweizerisches Strafgesetzbuch vom 21.12.1937 (StGB)

Art. 56 Abs. 3 Bundesgesetz über die Krankenversicherung vom 18.3.1994 (KVG) 


\section{Hinweise zur Ausarbeitung dieser Richtlinien}

Die vorliegenden Richtlinien der SAMW treten am 1. Februar 2013 in Kraft; sie ersetzen die analogen Richtlinien von 2006.

Mitglieder der für die Fassung 2006 verantwortlichen Arbeitsgruppe

Dr. Hermann Amstad, SAMW, Basel

Prof. Christoph Beglinger, Universitätsspital Basel

Prof. Jérôme Biollaz, Universitätsspital Lausanne

Dr. Max Giger, FMH, Winterthur

Dr. iur. Dieter Grauer, SGCI Chemie Pharma Schweiz, Zürich

Fürsprecher Hanspeter Kuhn, FMH, Bern

Prof. Urban Laffer, Regionalspital Biel

Prof. Thomas Lüscher, Universitätsspital Zürich

Dr. iur. Jürg Müller, Rechtsdienst, Universitätsspital Basel

lic.iur. Michelle Salathé, SAMW, Basel

Prof. Werner Stauffacher, SAMW, Basel

Dr. Urs Strebel, Kreisspital Männedorf

Mitglieder der auch für die Fassung 2012 verantwortlichen

Beratenden Kommission für die Umsetzung der Richtlinien

"Zusammenarbeit Ärzteschaft-Industrie»

Prof. Walter Reinhart, Chur (Vorsitz)

Dr. Gilbert Abetel, Orbe

Prof. Anne-Françoise Allaz, Genf

Dr. Hermann Amstad, Basel

Prof. Jerôme Biollaz, Lausanne

Dr. iur. Dieter Grauer, scienceindustries, Zürich

Prof. Hans-Rudolf Koelz, Uitikon Waldegg

Prof. Thomas Lüscher, Zürich

Dr. Christian Marti, Winterthur

Dr. Alain Michaud, Nyon

Dr. iur. Jürg Müller, Basel

Prof. Reto Obrist, Sierre

Dr. Gert Printzen, Luzern

Dr. Urs Strebel, Männedorf

Dr. Markus Trutmann, Biel

\section{Fachliche Beratung}

Dr. Peter Kleist, GlaxoSmithKline, Münchenbuchsee

\section{Genehmigung}

Genehmigt vom Senat der SAMW am 29. November 2012.

\section{Kontakt}

Schweizerische Akademie

der Medizinischen Wissenschaften

Petersplatz 13

$\mathrm{CH}-4051$ Basel

Tel. +41612699030

Fax +416126990 39

E-Mail mail@samw.ch

www.samw.ch 
Bestelladresse

SAMW

Petersplatz 13

CH-4051 Basel

Tel.: +4161269 9030

E-Mail: mail@samw.ch

\section{Gestaltung}

Howald Fosco, Basel

Druck

Kreis Druck, Basel

Auflage

$1200 \mathrm{D}, 600 \mathrm{~F}$

C SAMW 2013

(1) Die SAMW ist Mitglied der Akademien
der Wissenschaften Schweiz 


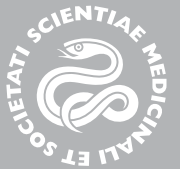

SAMW

Schweizerische Akademie

der Medizinischen

Wissenschaften

\section{ASSM}

Académie Suisse

des Sciences Médicales

\section{ASSM}

Accademia Svizzera delle

Scienze Mediche

\section{SAMS}

Swiss Academy

of Medical Sciences 